\title{
Ground-Penetrating Radar Antenna Modeling
}

\author{
Zhubo Huang, Kenneth Demarest, and Richard Plumb \\ University of Kansas, Radar Systems and Remote Sensing Laboratory \\ 2291 Irving Hill Road, Lawrence, Kansas 66045, USA \\ Tel: 913/864-7395 , FAX: 913/864-7789, E-mail: demarest@eecs.ukans.edu
}

\begin{abstract}
Detecting subsurface objects by using GroundPenetrating Radar (GPR) has received considerable interest in recent years. In order to interpret radar signals from buried objects, one must have the ability to model a large range of objects, grounds and radar antennas, theoretically or numerically, so that a real GPR system can be simulated. Many investigations have been done for modeling objects (scatterers) and grounds, but few have involved realistic antennas. This paper presents a technique to model real GPR antennas located above a ground in which an object is buried. Numerical results are presented to verify this technique.
\end{abstract}

\section{INTRODUCTION}

The increasing use of ground-penetrating radar systems has brought about the need for sophisticated GPR modeling tools that are capable of interpreting the radar returns from objects buried in a variety of grounds. The interpretation of radar returns involves several research subjects, such as the modeling of radar antennas, grounds and buried objects. The interpretation of radar returns can be helpful for understanding how to detect and identify buried objects with GPR systems.

A computer code that is used to simulate the performance of GPR systems is called a GPR simulator. In general, a good GPR simulator is capable of correctly modeling the characteristics of the antennas, the ground, and the scatterers. Unfortunately, techniques that are good at modeling one aspect of the problem are usually not well suited for others. For example, the finite-difference time-domain technique (FDTD) [1] is well suited for modeling dielectric spaces, such as grounds and scatterers, but is not well suited for modeling complex antennas. Conversely, the method of moments (MOM) [2] is well suited for modeling complex antennas, but not penetrable objects and real grounds. Since accurate ground modeling is usually essential, most GPR simulators concentrate on accurate ground-scatterer modeling at the expense of modeling complex antennas.

This paper presents a technique that is able to model complex antennas in the presence of real grounds and scatterers. This technique makes use of the well-known equivalence principle from electromagnetics, which allows the overall GPR geometry to be divided into two subgeometries. Different numerical techniques can then be used to model different aspects of the overall problem. An iterative procedure is used to link two sub-geometries to the actual geometry being modeled. This technique is described in the following section, along with numerical results.

\section{FORMULATION OF THE PROBLEM}

Consider the typical GPR geometry which is shown in Fig. 1. Here, an antenna is located above a ground in which an object (scatterer) is buried. The antenna transmits an incident field toward the ground. This field is partially transmitted into the ground and then scattered by the buried object.

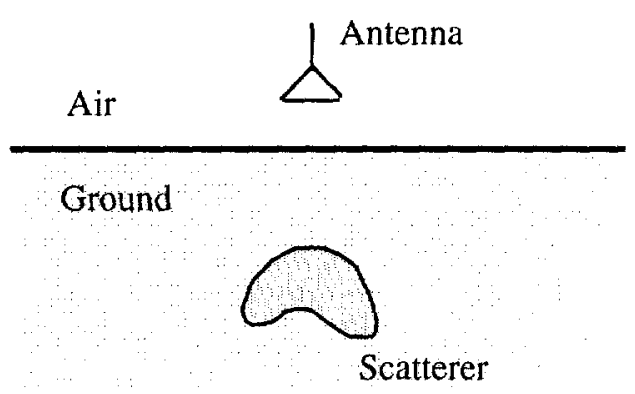

Figure 1. A typical ground-penetrating radar geometry.

Unfortunately, no one technique is well suited for modeling this typical GPR geometry. This is because there is an interaction between the antenna and the ground-scatterer, which requires the technique to model both antenna and ground-scatterer, as well as their interaction.

We can analyze this GPR geometry by using the Schelkunoff equivalence principle [3] to split the original geometry into two sub-geometries. This is accomplished by surrounding the antenna in Fig. 1 with a mathematical surface $S$ and removing either the interior elements or the exterior elements. The fields generated by these removed objects are supported by a set of equivalent electric and magnetic surface currents that are impressed on $S$. In this way, the complicated geometry can be divided into two subgeometries: an antenna geometry and a ground-scatterer geometry.

Fig. 2 shows these sub-geometries. The antenna geometry consists only of the antenna and the equivalent currents. In this geometry, the fields inside $S$ are identical to the fields inside $S$ in the original geometry, and zero outside $S$. The ground-scatterer geometry consists only of the ground, the 
scatterer, and the equivalent currents on $\mathrm{S}$. The fields outside $S$ in this sub-geometry are identical to those in the original geometry, and zero inside $S$. The equivalent surface currents that must be placed on $S$ in both sub-geometries are given by

$$
\begin{aligned}
\mathbf{J}_{\mathbf{s}} & =\mathbf{n} \times \mathbf{H} \\
\mathbf{M}_{\mathbf{s}} & =\mathbf{E} \times \mathbf{n}
\end{aligned}
$$

where $\mathbf{n}$ is the outwardly pointing unit vector of $S, \mathbf{J}_{\mathbf{s}}$ and $\mathbf{M}_{\mathbf{s}}$ are the electric and magnetic surface currents respectively, and $\mathbf{E}$ and $\mathbf{H}$ are the electric and magnetic fields on $S$ in the original geometry, respectively. The values of these surface currents can be determined by modeling the two subseparately and passing field information between them using an iterative procedure. This procedure models the interaction between the antenna and the ground-scatterer.

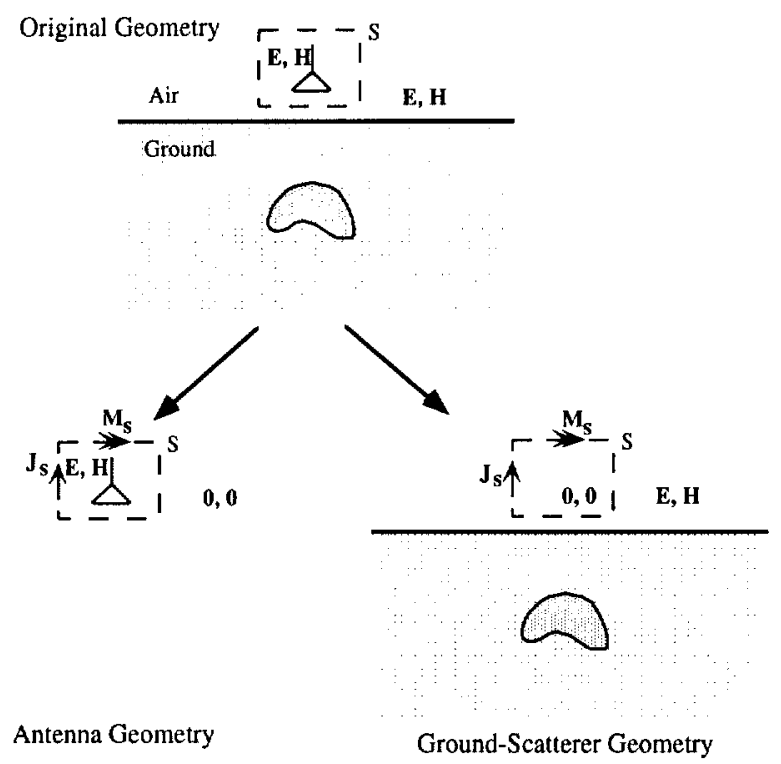

Figure 2. The geometry of the equivalent problem.

The iterative procedure starts by modeling the antenna geometry to get an initial estimate of surface currents on $S$. Then, one can impress these surface currents on $S$ to model the ground-scatterer geometry. By sampling the fields on $S$, one can then obtain an improved estimate of the surface currents that includes the initial currents and the currents due to the effect of the ground-scatterer. The next step is to model the antenna geometry again, while impressing the new surface currents on $S$. By sampling the fields on $S$, the initial surface currents on $S$ can be updated. These updated currents are impressed on $S$ to repeat modeling the groundscatterer geometry. A more accurate solution (surface current or field) can be obtained by performing more iterations.

Many numerical methods can be used for modeling each sub-geometry depending on the complexity of the problem.
For example, if the antenna is simple, such as a thin wire dipole, the FDTD method can be used for both subgeometries. In many GPR applications, the antenna usually is too complicated for the FDTD method to model. Therefore, in order to handle more complicated antennas, one can use the MOM to model the antenna and the FDTD to model the ground-scatterer geometry.

\section{SIMULATION}

In this section, a numerical experiment is presented to verify this iterative technique. The geometry of the experiment is shown in Fig. 3. Here, the radar antenna is selected as a thin wire dipole, driven by a voltage source. This antenna is simple enough so that the whole problem can be solved by either the FDTD method directly or by the iterative method. The result obtained by solving the whole problem by using the FDTD directly can be considered as an exact solution. Therefore, the iterative results can be compared with the exact result to verify the iterative technique.

In this experiment, the thin wire dipole (0.6-meter long) is located 0.4 meters above a ground. The ground is a stratified medium [4] with a relative dielectric constant that changes linearly from the surface (6.0) to 1.6 meters below the surface (8.0), with a uniform conductivity of $0.01 \mathrm{~s} / \mathrm{m}$. A perfectly conducting cube, 0.2 meter $^{3}$, is buried in the ground 0.6 meters below the ground surface. The exact solution is obtained in a $50 \times 50 \times 70$-cell FDTD space, in which the ground contains 40 cells in the vertical direction and the equivalence surface encloses $20 \times 10 \times 10$ cells. The FDTD cell size is 0.04 meters. The voltage source is a 6 -ns-doublepeak Gaussian pulse.

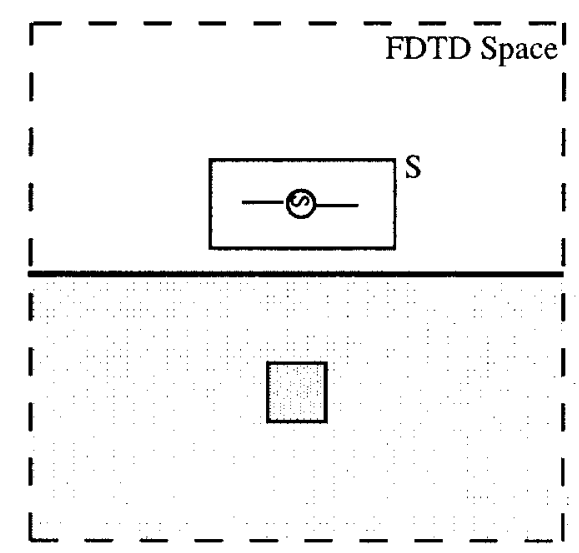

Figure 3. The geometry of the numerical experiment.

Fig. 4 shows the comparisons between the exact result (solid line) and iterative result (dashed line) when the antenna is excited with a voltage pulse. Here, the electric field 0.8 meters above the ground is shown. As can be seen, the difference between the exact result and the iterative result becomes smaller as more iterations are performed. The result of the fourth iteration is very close to the exact result. 
Fig. 5 shows the relative mean of absolute value of the scattered-field error. Here, the error field is the difference between the exact field and the iterative field. As can be seen in this figure, the relative mean error becomes smaller as more iterations are performed, indicating that the iterative procedure is capable of correctly modeling the antenna, the ground, and the scatterer.
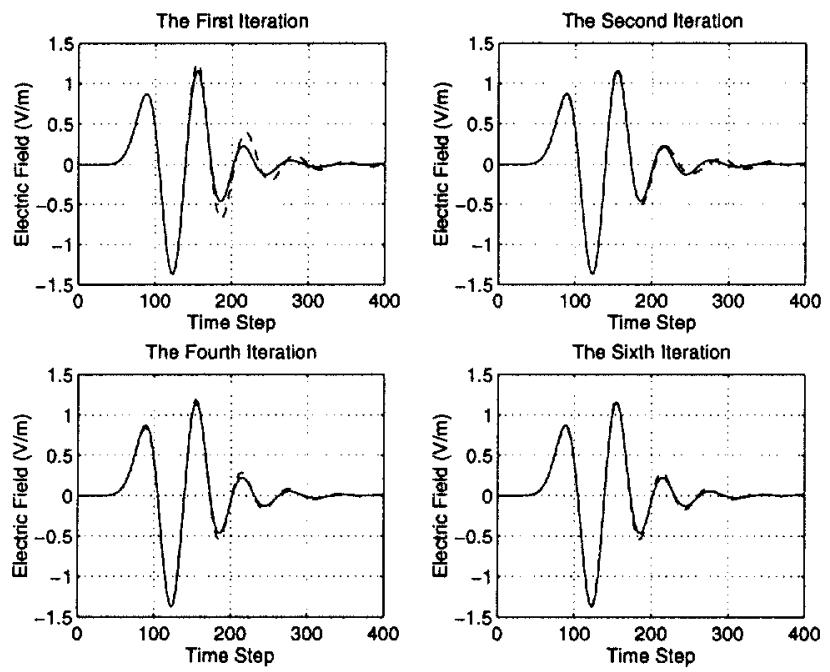

Figure 4: Electric field at one point above the ground.

\section{CONCLUSION}

In this paper we have presented an iterative technique that is capable of modeling complex antennas, grounds, and scatterers. This technique allows the antenna to be modeled separately from the ground-scatterer geometry, thus allowing different numerical techniques to model both parts of the overall geometry.

Although the numerical results presented here used the same numerical technique (FDTD) to model both parts of the overall problem, this is not a restriction of the overall technique itself.

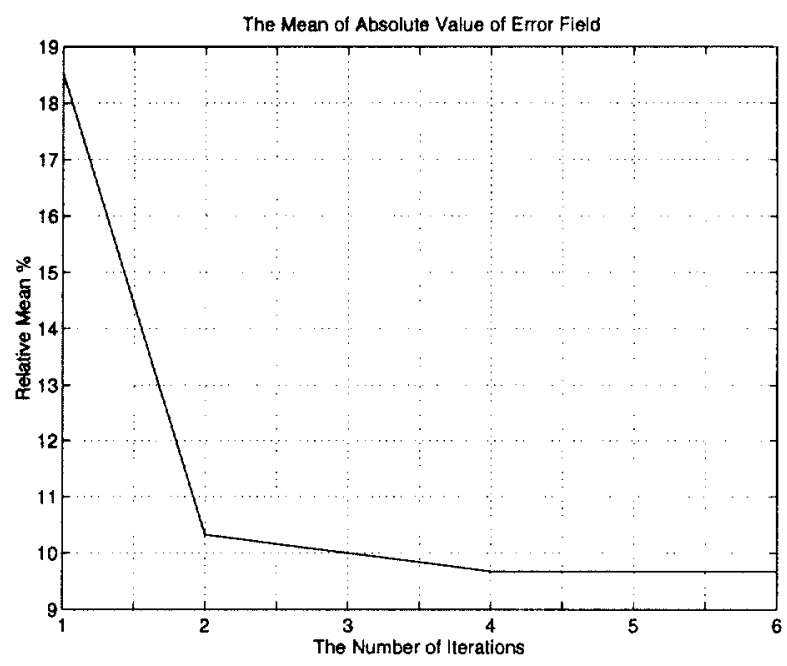

Figure 5: Relative mean of the error field.

\section{REFERENCES}

[1] K. S. Yee, "Numerical Solution of Initial Boundary Value Problems Involving Maxwell's Equations in Isotropic Media," IEEE Transaction on Antenna and Propagation, vol. AP-14, no. 3, pp. 302-307, May 1966.

[2] Roger F. Harrington, Field Computation by Moment Methods, New York, IEEE Press, 1993.

[3] Roger F. Harrington, Time-Harmonic Electromagnetic Fields, New York, McGraw-Hill Book Company, 1961.

[4] K. R. Demarest, R. G. Plumb, and Z. Huang, " FDTD Modeling of Scatterers in Stratified Media," IEEE Transaction on Antenna and Propagation, vol. AP-43, no. 10 , pp. 1164-1168, October 1995 . 